\title{
LAS ESTRATEGIAS FINANCIERAS Y EL PATRIMONIO DE LAS COOPERATIVAS DE AHORRO Y CRÉDITO EN UN CONTEXTO DE ECONOMIA POPULAR Y SOLIDARIA
}

\section{THE FINANCIAL STRATEGIES AND THE HERITAGE OF SAVINGS AND CREDIT COOPERATIVES IN A CONTEXT OF POPULAR AND SOLIDARITY ECONOMY}

\section{Recebimento: 31/07/2017- Aceite: 08/11/2017- Publicação: 23/12/2017 Processo de Avaliação: Double Blind Review}

Hermel David Ortíz Roman ${ }^{1}$

Magíster en Gerencia Empresarial

Economista

Facultad de Contabilidad y Auditoría - Universidad Técnica de Ambato hd.ortiz@uta.edu.ec

Mery Esperanza Ruiz Guajala

Magíster en Costos y Gestión Financiera

Economista

Facultad de Contabilidad y Auditoría - Universidad Técnica de Ambato meryeruiz@uta.edu.ec

Ana Graciela Molina Jimenez

Magíster en Auditoría Integral

Doctora en Contabilidad y Auditoría

Facultad de Contabilidad y Auditoría - Universidad Técnica de Ambato anagmolina@uta.edu.ec

Lissette Gancino-Ortega

Ingeniera en Contabilidad y Auditoría

Facultad de Contabilidad y Auditoría - Universidad Técnica de Ambato lissgancino@gmail.com

\section{RESUMEN}

En el presente trabajo de investigación se analiza las estrategias financieras y los incrementos patrimoniales de las cooperativas de ahorro y crédito del segmento dos del Cantón Ambato, para lo cual se decidió estudiar las estrategias financieras que tuvieron mayor impacto en el aumento del patrimonio, se aplicaron indicadores financieros para determinar si el patrimonio incremento durante el año 2015. Esta problemática se ve afectada en la rentabilidad de las

\footnotetext{
${ }^{1}$ Autor para correspondência: Universidad Técnica de Ambato- Av. Colombia y Chile, Ambato, Ecuador.
} 
cooperativas, reduciendo así su competitividad y capacidad para mantenerse en el mercado cooperativo. Uno de los indicadores más importantes es la suficiencia patrimonial que relaciona el patrimonio técnico constituido y los activos y contingentes ponderados por riesgo, en el cual la ley exige mantener un mínimo del $9 \%$ de este indicador para respaldar las operaciones actuales y futuras de las instituciones. Se analizó además si las cooperativas cumplen con la normativa establecida por el Código Orgánico Monetario y Financiero y la Ley de Economía Popular y Solidaria para el fortalecimiento del patrimonio, utilidades y fondo irrepartible de reserva legal. Se concluye que las estrategias financieras inciden significativamente en el patrimonio de las cooperativas, y se propone un modelo operativo para las estrategias financieras y el incremento del patrimonio el cual permitirá cumplir con todos los objetivos establecidos por cada entidad cooperativa.

PALABRAS CLAVE: Cooperativas Segmento Dos, Estrategias Financieras, Incrementos, Indicadores SEPS, Patrimonio.

\begin{abstract}
.
In the present research the financial strategies and the patrimonial increases of the savings and credit cooperatives of segment two of the Canton Ambato are analyzed, for which it was decided to study the financial strategies that obtained the greatest impact in the increase of the patrimony, financial indicators were applied to determine if the equity increased during the year 2015. This problem is affected in the profitability of cooperatives, thus reducing their competitiveness and ability to stay in the cooperative market. One of the most important indicators is the patrimonial sufficiency that relates the technical patrimony constituted and the assets and contingents weighted by risk, in which the law requires a minimum of 9\% of this indicator to be maintained to support the institution's current and future operations. It was also analyzed whether the cooperatives comply with the regulations established by the Monetary and Financial Organic Code and the Law of Popular and Solidarity Economy for the strengthening of patrimony, utilities and irreparable legal reserve fund. We conclude that financial strategies have a significant impact on the assets of cooperatives, and propose an operational model for the financial strategies and the increment of the patrimony that will allow to fulfill all the objectives established by each cooperative entity.
\end{abstract}

KEYWORDS: Cooperatives Segment Two, Financial Strategies, Increments, Seps Indicators, Patrimony. 


\section{INTRODUCCIÓN}

El cooperativismo es hoy uno de los grandes pilares sobre los que se permite el desenvolvimiento económico. En el Ecuador, se ha establecido un modelo económico como alternativa de desarrollo social y se ha constituido en un sector institucional estratégico para la economía popular y solidaria, con grandes posibilidades de crecimiento y fortalecimiento y es un gran referente para el desarrollo económico y el fomento de la integración social. Actualmente en el Ecuador existen aproximadamente 1.012 cooperativas divididas por provincias y segmentos según la Superintendencia de Economía Popular y Solidaria (SEPS, 2016), que han aportado significativamente a la producción y al empleo ecuatoriano.

En Tungurahua existen 165 cooperativas divididas en los cinco segmentos, de las cuales 4 pertenecen al segmento 2 según la (SEPS, 2016) y son la Cooperativa de Ahorro y Crédito Ambato Ltda., Indígena SAC Ltda., Kullki Wasi Ltda., y Chibuleo Ltda., que considerando el tamaño y los activos que poseen deben implementar estrategias financieras eficientes para un manejo adecuado de sus recursos. Las cooperativas desempeñan múltiples funciones que no sólo son económicas, sino también sociales, una cooperativa se diferencia de una organización comercial en que su principal objetivo es servir a sus integrantes, pero a la vez tiene un impacto positivo en la comunidad en la que opera.

En esta investigación se analizará las estrategias financieras implantadas por las Cooperativas de Ahorro y Crédito del segmento dos del Cantón Ambato que son de gran importancia para establecer si existen incrementos patrimoniales, podremos identificar si las estrategias financieras son las adecuadas para determinar variaciones en el patrimonio, además nos permitirá saber cómo se han desarrollado las cooperativas en los últimos dos años. Dentro de los planes estratégicos se establecen planes financieros que permite la evaluación de la situación financiera para determinar su crecimiento. Una estrategia clara y razonada es la clave de la administración para hacer negocios, su mapa para llegar a la ventaja competitiva y así mejorar su desempeño financiero (Thompson, Peteraf, Gamble, \& Strickland, 2012), es decir; que una estrategia bien definida ayuda al cumplimiento de los objetivos institucionales y crecimiento de la entidad.

Según el Código Orgánico Monetario y Financiero (COMF, 2014) artículo 190 establece que para las cooperativas de ahorro y crédito se debe mantener un patrimonio técnico de al menos el $9 \%$ con respecto a la suma ponderada por riesgo de sus activos y contingentes, lo que significa que si se obtienen resultados mayores a dicho porcentaje las cooperativas se encuentran financieramente bien.

Al analizar las dos variables de esta investigación se plantea un modelo operativo en el que se desarrollan actividades respecto a las estrategias y manejo del patrimonio para permitir de mejor manera el desempeño de cada cooperativa, se aplicará la estrategia que ha permitido obtener mayores incrementos para mejorar sus situación financiera y permanencia en el mercado cooperativo, de igual manera se aplicaron indicadores financieros que permiten medir el incremento del patrimonio, estos indicadores son establecidos por la SEPS en donde cada cooperativa debe reportar una información mensual con dichos indicadores. 


\section{REVISIÓN DE LA LITERATURA}

Es indispensable que las empresas tengan la capacidad para integrar y dirigir sus recursos orientados a la ejecución eficaz de la estrategia, lo que involucra procesos, personas, organización, cultura organizacional, sistemas de información, indicadores y retroalimentación (Robbins \& Coulter, 2010). La ejecución de la estrategia se convierte de este modo en un deber y compromiso no sólo de los directivos sino de todos los colaboradores que forman parte de la empresa (Olivares-Valentín, 2011). Así como en la planeación estratégica es indispensable el diseño claro y preciso de la estrategia como elemento central de un plan de negocios, aún más importante es que las empresas sean capaces de ejecutar con eficacia las estrategias y con ello alcanzar sus metas y objetivos propuestos.

El proceso de planeación estratégica es la herramienta principal que una empresa tiene que utilizar para transformarse en una organización competitiva, porque a través de ella es posible determinar claramente a dónde quiere ir, de qué manera se guiará, a partir de donde se encuentra y puede fijar los elementos necesarios para lograr su misión (Ramírez, 2013). Los planes sirven de guía para toda la organización y la planeación proporciona dirección a los gerentes y al resto de empleados, reduce la incertidumbre ya que obliga a los gerentes a ver el futuro, y al aplicar estrategias ayudan a direccionar de mejor manera a la empresa.

La condición financiera suele considerarse como la mejor medida de la posición competitiva y del atractivo general de una empresa para los inversionistas. Determinar las fortalezas y debilidades financieras de una organización es esencial para la formulación efectiva de estrategias (David Fred R, 2013).

Una estrategia clara y razonada es la receta de la administración para hacer negocios, su mapa para llegar a la ventaja competitiva, su plan para complacer a los clientes y así mejorar su desempeño financiero (Thompson, Peteraf, Gamble, \& Strickland, 2012). La estrategia como lo mencionan algunos autores básicamente sirve de base para el cumplimiento de objetivos, al analizar las fortalezas y debilidades financieras de cada organización ayudan a posicionarse de manera competitiva frente a las demás empresas y a cumplir con los planes financieros establecidos previamente para mejorar su desempeño financiero.

Los planes financieros a largo plazo forman parte de una estrategia integrada que, junto con los planes de producción y marketing, lleva a la empresa hacia metas estratégicas (Pascale, 2009). Estos planes a largo plazo consideran los desembolsos propuestos en activos fijos, actividades de investigación y desarrollo, acciones de marketing y desarrollo de productos, estructura del capital y fuentes importantes de financiamiento (Gitman \& Castro Gutiérrez, 2010). Las estrategias a largo plazo permiten planificar en el lapso de cinco a diez años obteniendo beneficios futuros, mediante las inversiones, el financiamiento en la estructura financiera y los resultados que ayudan a un constante crecimiento y competitividad dentro de cada organización.

Entre las posibles estrategias financieras a corto plazo existentes, conviene que nos detengamos a analizar la conocida como gestión de tesorería que depende sincronizar las 
corrientes de entradas y salidas monetarias de la empresa durante un plazo de tiempo evitando la aparición de excedentes (Cantalapiedra Arenas, 2004). En las estrategias a corto plazo se pueden administrar el dinero corriente de la empresa, mediante planes financieros previamente establecidos, estas estrategias se implantan en un periodo de uno a dos años.

El patrimonio es un título que denota el interés de los socios o accionistas en la empresa; representa los aportes iniciales de capital más la acumulación de reservas, superávit de capital y utilidades no distribuidas. Es la diferencia que se obtiene como resultado de restar los pasivos de los activos (Zapata Sánchez, 2011). El patrimonio como lo mencionan algunos autores es el capital aportado por los socios y financiado por terceras personas (Rivero, 2001), (Baena Toro, 2010) . El patrimonio busca obtener el máximo beneficio posible favoreciendo a los socios, aquí interviene la creación de valor sobre las aportaciones que realizan los socios, solo así se podrá definir con claridad si la empresa está generando valor sobre cada aporte.

La capacidad de una empresa para obtener utilidades depende de la eficiencia y eficacia de sus operaciones, así como de los recursos que disponga. Por tanto, el análisis de rentabilidad se concentra principalmente en la relación entre los resultados de las operaciones, según se presentan en el estado de resultados, y los recursos disponibles para la empresa, que aparecen en el balance general (Warren, Reeve, \& Fess, 2005).

Uno de los indicadores que se aplicó en este proyecto de investigación es la suficiencia patrimonial mide la proporción de patrimonio efectivo frente a los activos inmovilizados, una mayor cobertura patrimonial de activos significa una mejor posición (Superintendencia de Bancos). La vulnerabilidad del patrimonio como lo menciona la superintendencia de bancos mide el riesgo del patrimonio frente a la cartera que no genera intereses y cartera vencida (Superintendencia de Bancos). En este trabajo de investigación se realizará el cálculo de dos años $(2014$ - 2015) por lo que nos permite controlar si la vulnerabilidad de un año a otro ha aumentado o disminuido, siendo lo ideal disminuir el porcentaje de dicho indicador, lo que significaría que la cartera improductiva estaría reduciendo, ya sea en créditos vencidos o que no generan intereses.

La rentabilidad del patrimonio expresa el comportamiento de la situación económica y su retribución al capital propio proporcionando información de la entidad y su capacidad para autofinanciar actividades ordinarias (German, 2006). Por lo tanto el rendimiento sobre el patrimonio que se va aplicar medirá el valor que está generando sobre su capital propio, es decir, mide la tasa de crecimiento sobre las ganancias de las cooperativas.

En el artículo 190 del Código Orgánico Monetario y Financiero la solvencia patrimonial se obtiene del patrimonio técnico constituido que éste a su vez se obtiene de la suma del patrimonio técnico primario compuesto del capital social y la reserva legal, y del patrimonio técnico secundario compuesto de la reserva facultativa, estatutaria y los resultados. Por otro lado los activos y contingentes ponderados por riesgo se componen del total de activos menos los fondos disponibles (COMF, 2014). 
Todos los indicadores mencionados anteriormente son aplicados en las cooperativas de ahorro y crédito del segmento dos del cantón Ambato, aplicando en los estados financieros de los años 2014 y 2015.

\section{METODOLOGÍA}

La investigación de campo se caracteriza principalmente por la recolección de información netamente en el lugar donde se desarrolla el problema. La investigación de campo es el estudio sistemático de los hechos en el lugar en que se producen los acontecimientos. En esta modalidad el investigador toma contacto en forma directa con la realidad, para obtener información de acuerdo con los objetivos del proyecto (Herrera, Medina, \& Naranjo, 2004). Esta modalidad es la más efectiva y utilizada al momento de realizar investigaciones, en nuestro caso se contará con información de cada una de las cooperativas del segmento dos del cantón Ambato, que ayudarán a sustentar el trabajo de investigación.

La investigación bibliográfica es parte del proceso de una investigación científica. Es la que se la realiza en los libros o textos (Cedeño Loor, 2010). La investigación bibliográfica hace referencia a las investigaciones de otros autores en donde procesan la información para dejarla como referencia para los futuros proyectos, estas investigaciones son de carácter científico, que son aprobados por las autoridades competentes para declarar su confiabilidad.

La investigación correlacional se basa específicamente en relacionar las dos variables que son objeto de estudio, el objetivo es estudiarlas y saber si se relacionan entre sí, es la que investiga la relación entre dos variables de un mismo hecho o fenómeno. Así se llega a determinar en qué medida un factor o factores de una variable modifica o varía los factores de la otra variable (Cedeño Loor, 2010).

El universo o población es un grupo de personas o cosas similares en uno o varios aspectos, que forman parte del objeto de estudio (Eyssautier de la Mora, 2002). La población que se define en este proyecto de investigación son las 4 Cooperativas de Ahorro y Crédito pertenecientes al cantón Ambato segmento dos, y los documentos que se procederán analizar son los Estados Financieros y los Planes Estratégicos que ayudarán a esta investigación. A continuación se describe la población total a utilizar:

Revista ENIAC Pesquisa, Guarulhos (SP), V.6, n.2, jun.- dez. 2017 
Tabla 1. Población

\begin{tabular}{ll}
\hline \multicolumn{1}{c}{ Cooperativas } & $\mathrm{N}^{\circ}$ \\
\hline Cooperativa de Ahorro y Crédito Ambato Ltda. & 1 \\
Cooperativa de Ahorro y Crédito Chibuleo Ltda. & 1 \\
Cooperativa de Ahorro y Crédito Indígena Sac Ltda. & 1 \\
Cooperativa de Ahorro y Crédito Kullki Wasi Ltda. & 1 \\
\hline Total: & 4 \\
\hline
\end{tabular}

Fuente: COACS del Segmento dos Cantón Ambato

Elaborado por: Lissette Alejandra Gancino Ortega

La muestra es la parte de la población que se selecciona, de la cual realmente se obtiene la información para el desarrollo del estudio y sobre la cual se efectuarán la medición y la observación de las variables objeto de estudio (Bernal Torres, 2006). En nuestra investigación no se utilizó el cálculo de la muestra debido a que la población que es el objeto de estudio es pequeña, por lo tanto se trabajó con su totalidad.

\section{RESULTADOS}

\section{Principales resultados de las estrategias financieras}

Se ha realizado una lista de chequeo que constan de 16 preguntas para cada variable a evaluar en este proyecto de investigación dentro de las Cooperativas de Ahorro y Crédito del segmento dos pertenecientes al Cantón Ambato, para lo cual se procedió a evaluar las estrategias financieras que aplican estas cooperativas para determinar incrementos patrimoniales.

Los principales resultados se analizan a continuación mediante una tabla en donde se determinan solo las preguntas más relevantes en la investigación:

Revista ENIAC Pesquisa, Guarulhos (SP), V.6, n.2, jun.- dez. 2017 
Tabla 2. Resultados Lista de Chequeo Estrategias Financieras

Estrategias Financieras

\begin{tabular}{|c|c|c|c|c|c|}
\hline \multirow[b]{2}{*}{$\mathrm{N}^{\circ}$} & \multirow[b]{2}{*}{ Pregunta } & \multicolumn{4}{|c|}{ Cooperativa de Ahorro y Crédito } \\
\hline & & $\begin{array}{l}\text { Ambato } \\
\text { Ltda. }\end{array}$ & $\begin{array}{l}\text { Chibuleo } \\
\text { Ltda. }\end{array}$ & $\begin{array}{l}\text { Indígena Sac } \\
\text { Ltda. }\end{array}$ & $\begin{array}{c}\text { Kullki } \\
\text { Wasi Ltda. }\end{array}$ \\
\hline 1 & $\begin{array}{lll}\text { ¿La cooperativa } & \text { establece } \\
\text { periódicamente estrategias } & \text { para } & \text { el } \\
\text { incremento patrimonial? } & & \end{array}$ & SI & SI & SI & NO \\
\hline 2 & $\begin{array}{l}\text { ¿La cooperativa aplica indicadores } \\
\text { financieros para la evaluación de las } \\
\text { estrategias establecidas para la toma de } \\
\text { decisiones? }\end{array}$ & SI & SI & SI & SI \\
\hline 3 & $\begin{array}{l}\text { ¿La cooperativa destina el } 50 \% \text { de las } \\
\text { utilidades para el Fondo Irrepartible de } \\
\text { Reserva Legal como lo estipula el } \\
\text { artículo } 50 \text { de la Ley de Economía } \\
\text { Popular y Solidaria? }\end{array}$ & SI & SI & SI & SI \\
\hline 4 & $\begin{array}{l}\text { ¿Por cada crédito que realizan los } \\
\text { socios en la cooperativa se destina un } \\
\text { porcentaje para certificados de } \\
\text { aportación? }\end{array}$ & NO & SI & NO & NO \\
\hline 5 & $\begin{array}{l}\text { ¿Existe un monto límite de certificados } \\
\text { de aportaciones para los socios que han } \\
\text { efectuado varios créditos? }\end{array}$ & NO & NO & NO & NO \\
\hline 6 & $\begin{array}{l}\text { ¿La cooperativa ha determinado cual es } \\
\text { el porcentaje de Patrimonio Técnico? }\end{array}$ & SI & SI & SI & SI \\
\hline 7 & $\begin{array}{l}\text { ¿La cooperativa cumple con el artículo } \\
190 \text { del Código Orgánico Monetario y } \\
\text { Financiero en donde menciona que } \\
\text { deben mantener un patrimonio técnico } \\
\text { de al menos el } 9 \% \text { ? }\end{array}$ & SI & SI & SI & SI \\
\hline 8 & $\begin{array}{l}\text { ¿Para incrementar el capital la } \\
\text { cooperativa ocasionalmente realiza } \\
\text { promociones para elevar los } \\
\text { certificados de aportación? }\end{array}$ & NO & NO & SI & SI \\
\hline
\end{tabular}

Nota: Se han tomado solo las preguntas más relevantes de la investigación. Fuente: Lissette Alejandra Gancino Ortega

Revista ENIAC Pesquisa, Guarulhos (SP), V.6, n.2, jun.- dez. 2017 


\section{Análisis e Interpretación}

De las cuatro cooperativas evaluadas tres establecen estrategias periódicamente dentro de la Planificación Estratégica que se elabora anualmente para los incrementos patrimoniales, la cooperativa Kullki Wasi Ltda., no establece estrategias ya que las leyes y normativas implantadas por la SEPS les ayuda con parámetros para incrementar su patrimonio.

Todas las cooperativas aplican indicadores financieros para la evaluación de las estrategias establecidas que forman parte de la toma de decisiones dentro de las empresas, ya que todo lo que se mide puede ser evaluado, para determinar si las estrategias que se aplican son efectivas. Por otro lado, también todas han obtenido utilidad en los últimos años permitiéndoles incrementar la totalidad de su patrimonio.

Las cooperativas de este segmento cumplen con el artículo 50 de la Ley de Economía Popular y Solidaria en donde menciona que se destinará el 50\% de las utilidades para el Fondo Irrepartible de Reserva Legal que ayudará para solventar contingencias patrimoniales, además menciona que este fondo no podrá ser distribuido entre los socios.

La cooperativa Chibuleo Ltda., por cada crédito que concede destina un porcentaje para certificados de aportación dependiendo de los montos de los créditos, la cooperativa ha realizado una propuesta a la SEPS en donde se establece un cuadro base y según los montos de los créditos cobrar hasta el 3\% para incrementar los certificados de aportación. Por otro lado las tres cooperativas cobran en cada crédito hasta el 3\% que se realiza para el fortalecimiento del fondo irrepartible de reserva legal, cabe mencionar que las cooperativas que mantengan un patrimonio técnico de al menos el 9\% según lo determina el artículo 190 del Código Orgánico Monetario y Financiero, podrá decidir el porcentaje que cobran para el fortalecimiento de la reserva legal siempre que no exceda del $3 \%$.

Ninguna cooperativa ha establecido un monto límite para certificados de aportación por cada crédito que realizan los socios. La cooperativa Chibuleo Ltda., establece un monto de $\$ 2.000,00$ para lo cual se está implementando en sus estatutos dicha política. Por lo tanto, si las cooperativas no establecen un monto límite, las personas que realicen varios créditos mantendrán en certificados de aportación un valor elevado y provocara que algunos socios liquiden sus cuentas en dichas entidades.

El artículo 190 del Código Orgánico Monetario y Financiero menciona que las cooperativas deben mantener constantemente una relación entre su patrimonio técnico y la suma ponderada por riesgo de sus activos y contingentes de al menos el $9 \%$. La cooperativa Ambato Ltda., ha determinado un $16.07 \%$ en su patrimonio técnico del año 2015; la cooperativa Chibuleo Ltda., obtiene un $15.73 \%$ en el año 2015; la cooperativa Indígena Sac Ltda., obtuvo un $9.77 \%$ en el año 2015 y la cooperativa Kullki Wasi Ltda., determinó un $14.44 \%$ para el 2015. Por lo tanto todas las cooperativas analizadas cumplen con este artículo manteniendo un patrimonio técnico mayor al 9\%. 
La cooperativa Indígena Sac Ltda., y Kullki Wasi Ltda., realizan promociones para que sus socios completen sus certificados de aportación, además la cooperativa Indígena Sac Ltda., ha realizado rifas para los socios inactivos y para actualización de datos, esto permite incrementar su patrimonio. Por otro lado, la cooperativa Ambato Ltda., y Chibuleo Ltda., no realizan promociones ocasionales ya que alcanzan un patrimonio técnico elevado y no consideran necesario realizar dichas promociones.

\section{Principales resultados del patrimonio}

En esta segunda parte de la lista de chequeo se analizó el patrimonio mediante indicadores implantados por la SEPS para determinar los incrementos patrimoniales de las Cooperativas de Ahorro y Crédito del Segmento dos pertenecientes la Cantón Ambato.

Revista ENIAC Pesquisa, Guarulhos (SP), V.6, n.2, jun.- dez. 2017 
Tabla 3. Resultados Lista de Chequeo Patrimonio

\begin{tabular}{|c|c|c|c|c|c|c|c|c|c|}
\hline \multicolumn{10}{|c|}{ Patrimonio } \\
\hline \multirow[b]{2}{*}{$\mathrm{N}^{\circ}$} & \multirow[b]{2}{*}{ Pregunta } & \multicolumn{8}{|c|}{ Cooperativa de Ahorro y Crédito } \\
\hline & & \multicolumn{2}{|c|}{ Ambato Ltda. } & \multicolumn{2}{|c|}{ Chibuleo Ltda. } & \multicolumn{2}{|c|}{$\begin{array}{l}\text { Indígena Sac } \\
\text { Ltda. }\end{array}$} & \multicolumn{2}{|c|}{$\begin{array}{l}\text { Kullki Wasi } \\
\text { Ltda. }\end{array}$} \\
\hline 1 & $\begin{array}{l}\text { total con relación al año } \\
\text { anterior incrementó? }\end{array}$ & NO & $-7,59 \%$ & SI & $19,96 \%$ & SI & $7,65 \%$ & SI & $25,50 \%$ \\
\hline 2 & $\begin{array}{l}\text { ¿Existe crecimiento de las } \\
\text { aportaciones de los socios con } \\
\text { respecto al año anterior? }\end{array}$ & NO & $-21,70 \%$ & SI & $24,01 \%$ & SI & $23,15 \%$ & SI & $18,66 \%$ \\
\hline 3 & $\begin{array}{l}\text { ¿Las utilidades del ejercicio } \\
\text { incrementaron con respecto a } \\
\text { las del año anterior? }\end{array}$ & NO & $-66,71 \%$ & SI & $0,26 \%$ & NO & $-43,39 \%$ & $\mathrm{NO}$ & $-53,55 \%$ \\
\hline 4 & $\begin{array}{l}\text { ¿Existe un aumento en la } \\
\text { suficiencia patrimonial del año } \\
2015 \text { de la cooperativa con } \\
\text { respecto al año 2014? }\end{array}$ & SI & $1,56 \%$ & NO & $-5,66 \%$ & NO & $-71,52 \%$ & NO & $-15,57 \%$ \\
\hline 5 & $\begin{array}{c}\text { ¿La vulnerabilidad del } \\
\text { patrimonio del año } 2015 \\
\text { (cartera improductiva / } \\
\text { patrimonio + resultados) creció } \\
\text { con relación al año } 2014 ?\end{array}$ & NO & $-92,79 \%$ & NO & $-2,54 \%$ & SI & $5,55 \%$ & SI & $38,50 \%$ \\
\hline 6 & $\begin{array}{l}\text { ¿El rendimiento del patrimonio } \\
\text { (ROE) incremento con relación } \\
\text { al año anterior (resultados del } \\
\text { ejercicio / patrimonio } \\
\text { promedio)? }\end{array}$ & NO & $-66,65 \%$ & NO & $-8,84 \%$ & NO & $-45,47 \%$ & NO & $-58,80 \%$ \\
\hline 7 & $\begin{array}{l}\text { ¿La solvencia patrimonial } \\
\text { (patrimonio técnico } \\
\text { constituido/activos } \\
\text { contingentes ponderados por } \\
\text { riesgo) del año } 2015 \text { aumentó } \\
\text { con relación al año 2014? }\end{array}$ & SI & $53,67 \%$ & SI & $40,86 \%$ & SI & $19,36 \%$ & SI & $6,39 \%$ \\
\hline 8 & $\begin{array}{l}\text { ¿El superávit por valuación de } \\
\text { propiedades, equipo y otros del } \\
2015 \text { incrementó con relación al } \\
\text { año anterior? }\end{array}$ & $\begin{array}{r}\text { Se } 1 \\
\mathrm{a}\end{array}$ & $\begin{array}{l}\text { ntiene de } \\
\text { a año }\end{array}$ & $\begin{array}{l}\text { No s } \\
\text { en } n\end{array}$ & $\begin{array}{l}\text { estableció } \\
\text { agún año }\end{array}$ & $\begin{array}{l}\text { Se } \mathrm{m} \\
\mathrm{añ}\end{array}$ & $\begin{array}{l}\text { antiene de } \\
\text { o a año }\end{array}$ & SI & $319,91 \%$ \\
\hline
\end{tabular}

Revista ENIAC Pesquisa, Guarulhos (SP), V.6, n.2, jun.- dez. 2017 
Tabla 5. (Continuación)

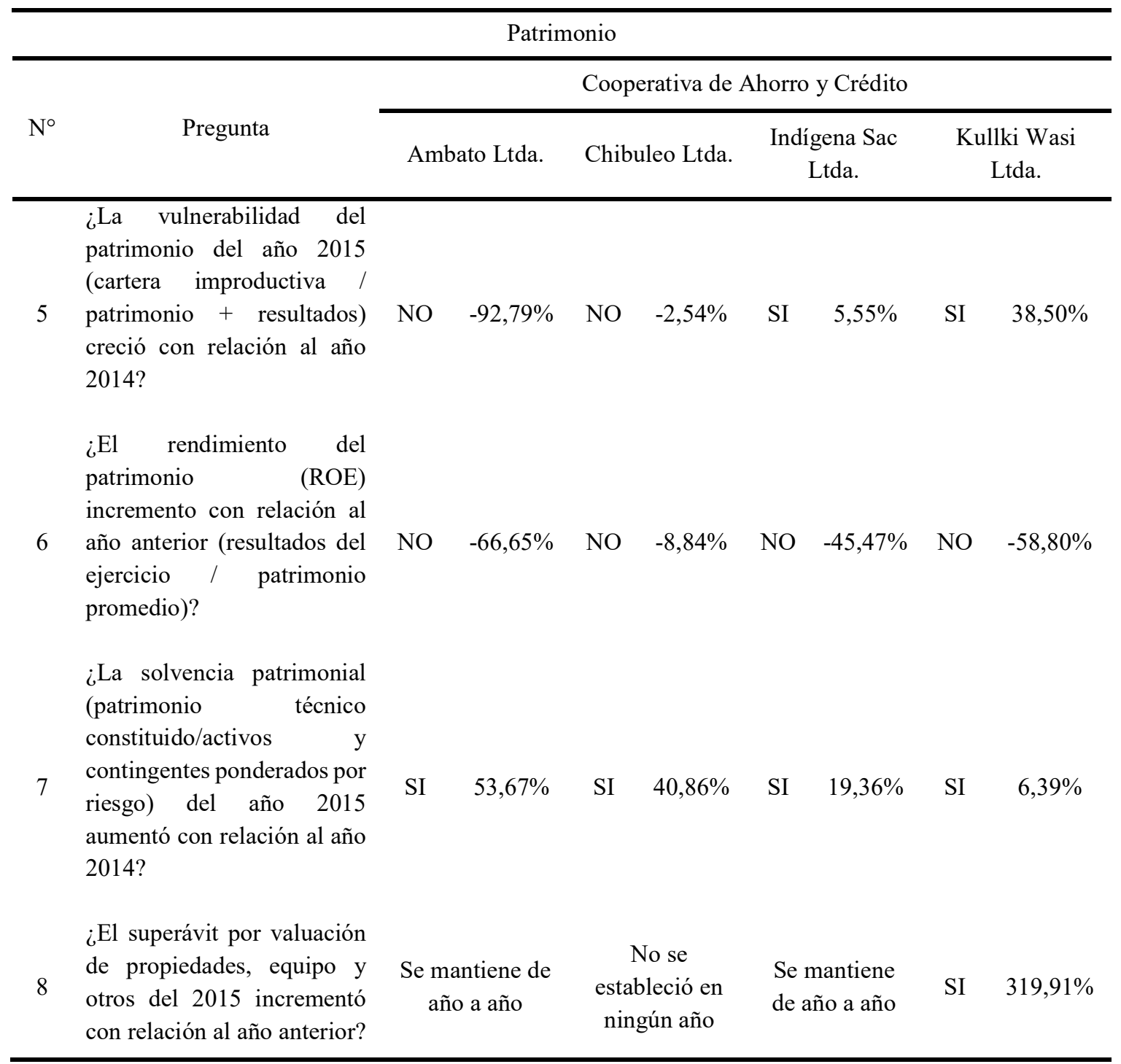

Nota: Se han tomado solo las preguntas más relevantes de la investigación.

Fuente: Lissette Alejandra Gancino Ortega

\section{Análisis e Interpretación}

El patrimonio total del año 2015 disminuyó en un 7.59\% en la cooperativa Ambato Ltda., debido a que sus utilidades decayeron; un 19.96\% en la cooperativa Chibuleo Ltda., el cual su valor más representativo fue en la reserva especial en donde mantienen los aportes para futuras capitalizaciones; un incremento del 7.65\% en la cooperativa Indígena Sac Ltda., a causa de las aportaciones de los socios que reflejan mayores incrementos; y un $25.50 \%$ en la cooperativa Kullki Wasi Ltda., con respecto al año 2014; es decir, que esta cooperativa

Revista ENIAC Pesquisa, Guarulhos (SP), V.6, n.2, jun.- dez. 2017 
aumento su patrimonio significativamente, dentro del cual el porcentaje más representativo fue la partida de superávit por valuación de propiedad planta y equipo.

Las aportaciones de los socios del año 2015 disminuyeron con relación al año 2014 en un $21.70 \%$ en la cooperativa Ambato Ltda. En la cooperativa Chibuleo Ltda., incrementó un $24.01 \%$ siendo este el valor más alto de las cuatro cooperativas analizadas, esto se debió a las estrategias que implantan para incrementar el patrimonio, ya que ellos cobran un porcentaje en los créditos que va a formar parte directamente del capital. La cooperativa Indígena Sac Ltda., y Kullki Wasi Ltda., incrementaron un 23.15\% y 18.66\% respectivamente, debido a las aportaciones que se realizan para la reserva legal en cada crédito otorgado.

En la cooperativa Ambato Ltda. las utilidades decayeron un $66.71 \%$ debido a que en año 2015 se obtuvo una pérdida de \$2'780.922,29 el cual redujo la utilidad del ejercicio de manera significativa. Por otro lado la cooperativa Chibuleo Ltda., incrementó su utilidad un $0.26 \%$ siendo este un valor favorable comparado con las otras cooperativas, para lo cual significa que la cooperativa obtuvo más ingresos en el 2015. La cooperativa Indígena Sac Ltda., disminuyó sus utilidades un $43.39 \%$ debido a que las utilidades del ejercicio también decayeron. En la cooperativa Kullki Wasi Ltda., las utilidades del año 2015 también redujeron un $53.55 \%$ con relación al año 2014 .

no de los indicadores implantado por la SEPS es la Suficiencia Patrimonial, el cual mide la proporción del patrimonio efectivo frente a los activos inmovilizados; es decir, los activos que no generan intereses. La cooperativa Ambato Ltda., ha incrementado este indicador en $1.56 \%$, es decir, esta cooperativa si ha cubierto sus activos con el patrimonio que posee, dentro de los dos año ha ido mejorando esta suficiencia. La cooperativa Chibuleo Ltda., disminuyó la suficiencia patrimonial un 5.66\%, la cooperativa Indígena Sac Ltda., disminuyó un $71.52 \%$ y la cooperativa Kullki Wasi disminuyó un $15.57 \%$; es decir que estas tres cooperativas si lograron cubrir sus activos en los dos últimos años, pero no incrementaron esta suficiencia ya que a mayor cobertura patrimonial de activos significa una mejor posición.

La vulnerabilidad del patrimonio en la cooperativa Ambato Ltda., y Chibuleo Ltda., disminuyó en $92.79 \%$ y $2.54 \%$ respectivamente, lo cual significa que para el año 2015 este índice se cumplió; esto significa que el patrimonio puede ir cubriendo la cartera que no genera intereses. Mientas que en el cooperativa Indígena Sac Ltda., y Kullki Wasi Ltda., la vulnerabilidad incrementó en $5.55 \%$ y $38.50 \%$ respectivamente; es decir, que este índice no ha cubierto la cartera improductiva en su totalidad en el año 2015.

Otro de los indicadores que se aplicaron es el ROE que mide la rentabilidad del patrimonio. La cooperativa Ambato Ltda., obtuvo una disminución de $66.65 \%$ en la rentabilidad; la cooperativa Chibuleo Ltda., disminuyó en $8.84 \%$; la cooperativa Indígena Sac Ltda., disminuyó en 45.47\%; la cooperativa Kullki Wasi Ltda., disminuyó en 58.80\%; es decir, que en los dos años si se obtuvo rentabilidad sobre su patrimonio pero en el año 2015 bajo comparado con el resultado obtenido en el 2014. 
La Solvencia Patrimonial es la suficiencia patrimonial que deben mantener constantemente las entidades para respaldar las operaciones actuales y futuras, y cubrir las pérdidas no protegidas por las provisiones de los activos de riesgo. La cooperativa Ambato ha obtenido un incremento del 53.67\%, la cooperativa Chibuleo Ltda., incrementó $40.86 \%$, la cooperativa Indígena Sac Ltda., aumentó $19.36 \%$ y la cooperativa Kullki Wasi Ltda. $6.39 \%$; es decir que las cuatro cooperativas lograron mantener la solvencia y suficiencia patrimonial mayor al 9\% como lo establece la SEPS.

Otro de los indicadores que se aplicó es el incremento del superávit por valuación de propiedades, equipo y otros, solo la cooperativa Kullki Wasi Ltda., incremento este indicador en un $319.91 \%$ lo que significa que este superávit es establecido por la SEPS para que se incremente su patrimonio legalmente cada 5 años, es decir, los incrementos que ha obtenido esta cooperativa se deben a mayor parte a los incrementos del superávit que exige la ley. La cooperativa Ambato e Indígena Sac Ltda., han mantenido este valor durante el 2014 y 2015. Por otro lado la cooperativa Chibuleo Ltda., no ha establecido esta partida en ningún año.

\title{
5. DISCUSIÓN
}

Según (Olivares-Valentín, 2011) en su investigación, Ejecución de la estrategia: Clave para el éxito empresarial define:

\begin{abstract}
Es indispensable que las empresas tengan la capacidad para integrar y dirigir sus recursos orientados a la ejecución eficaz de la estrategia, lo que involucra procesos, personas, organización, cultura organizacional, sistemas de información, indicadores y retroalimentación. La ejecución de la estrategia se convierte de este modo en un deber y compromiso no sólo de los directivos sino de todos los colaboradores que forman parte de la empresa (p. 28).
\end{abstract}

Así como en la planeación estratégica es indispensable el diseño claro y preciso de la estrategia como elemento central de un plan de negocios, aún más importante es que las empresas sean capaces de ejecutar con eficacia las estrategias y con ello alcanzar sus metas y objetivos propuestos; al aplicar procesos en la entidad que implique conocimiento, difusión, dominio y retroalimentación ayudan a lograr la ejecución de estrategias eficaces, además la participación de todas las personas, procesos e indicadores son de gran importancia para el cumplimiento de la estrategia

En la investigación de Estrategias financieras en la pequeña y mediana empresa de (Mariana Álvarez y J. L. Abreu, 2008) menciona que:

Para la determinación de estrategias financieras se deben primero que nada identificar las áreas claves en las que nos vamos a enfocar, que en este caso son: el director financiero, la competitividad, la innovación, la financiación y el riesgo financiero, la información financiera y los estados financieros, luego se deberá establecer un objetivo estratégico que deseemos alcanzar (p. 75). 
Al establecer áreas claves en las instituciones antes de la determinación de los objetivos estratégicos son de vital importancia ya que estaremos priorizando las áreas administrativas y financieras que impliquen el desarrollo de la entidad, además aplicar estrategias ayudarán a prevenir los riesgos financieros existentes. Las estrategias financieras correctamente aplicadas en las instituciones definen su éxito empresarial; por lo tanto se fortalecen y ayudan a su crecimiento de manera acelerada utilizando sus recursos adecuadamente.

\section{CONCLUSIONES}

Una vez analizadas las estrategias financieras de las cooperativas se determinó que tienen una gran incidencia en los incrementos patrimoniales de las cooperativas del segmento dos. Todas las cooperativas aplican indicadores financieros para la evaluación de estrategias y toma de decisiones, las cooperativas si cumplen con la normativa establecida por el Código Orgánico Monetario y Financiero para el fortalecimiento del patrimonio, utilidades y fondo irrepartible de reserva legal, además cada cooperativa decide enviar el total de las utilidades a la cuenta de aportes para futuras capitalizaciones, han optado por no distribuir las utilidades.

Una de las estrategias aplicadas que han tenido mayor incidencia para el incremento del patrimonio y que es utilizada por la cooperativa Chibuleo es el aporte de los socios que consiste en destinar un porcentaje para los certificados de aportación por cada crédito concedido. Las cooperativas analizadas no mantienen en sus políticas un límite en certificados de aportación para socios que realizan varios créditos, provocando insatisfacción en los socios, ya que por cada crédito estarían aportando en certificados cantidades representativas, dichos fondos no pueden ser disponibles para los mismos, provocando una masiva liquidación de socios.

Al analizar el patrimonio se puede concluir que todas las cooperativas incrementaron dicho patrimonio de un año a otro. La cooperativa Chibuleo Ltda., incrementó un $19.96 \%$ debido a la estrategia que implementaron, la cooperativa Kullki Wasi Ltda., incrementó un $25.50 \%$ su patrimonio debido al superávit por valuación de propiedades que se incrementa por ley cada cinco años. La cooperativa Indígena Sac Ltda., incrementó su patrimonio un 7.65\% debido a las aportaciones que mantiene. La cooperativa Ambato Ltda., disminuyó su patrimonio de un año a otro un $7.59 \%$ debido a disminuyeron las utilidades en el 2015.

La suficiencia patrimonial es un indicador que evalúa la proporción del patrimonio efectivo frente a los activos que no generan intereses. La cooperativa Ambato Ltda., fue la única que incrementó su suficiencia en un $1.56 \%$ comparado con los dos años analizados, este indicador refleja que todas las cooperativas tienen un porcentaje de suficiencia aceptable pero que no se ve incrementado de un año a otro. La vulnerabilidad del patrimonio evalúa el porcentaje obtenido de la cartera improductiva frente al patrimonio total, para lo cual se puede concluir que las cooperativas Ambato y Chibuleo mantienen un resultado positivo ya que la vulnerabilidad no creció de un año a otro, mientras que las cooperativas Indígena Sac y Kullki Wasi incrementaron esta vulnerabilidad. 
El ROE evaluado en las cooperativas determina que ninguna cooperativa obtuvo un incremento de dicho indicador, lo cual significa que todas las cooperativas obtienen buenos resultados en sus utilidades pero no existieron mejoras para el 2015. La solvencia patrimonial evalúa la relación que existe entre el patrimonio técnico constituido y los activos y contingentes ponderados por riesgo, para lo cual se ha determinado que todas las cooperativas obtuvieron un patrimonio técnico constituido mayor al $9 \%$ que señala la ley y debido a esto las cooperativas incrementaron la solvencia patrimonial.

\section{REFERENCIAS}

Baena Toro, D. (2010). Análisis Financiero. Colombia: Ecoe Ediciones.

Bernal Torres, C. A. (2006). Metodología de la Investigación. México: Pearson Educación.

Cantalapiedra Arenas, M. (2004). Cómo gestionar la relación de su empresa con los bancos. Madrid: Fundación Confemetal.

Cedeño Loor, R. (2010). Investigación Científica y Diseño de Tesis. Ecuador : Editorial Mar Abierto.

COMF. (12 de Septiembre de 2014). Código Orgánico Monetario y Financiero. Obtenido de http://www.asambleanacional.gob.ec/es/system/files/ro_codigo_organico_monetari o_y_financiero.pdf

David Fred R. (2013). Conceptos de Administración Estratégica. México: Pearson Educación.

Eyssautier de la Mora, M. (2002). Metodología de la Investigación . México: ECAFSA Thomson Learning.

German, H. (2006). Contabilidad Creativa. Argentina: ULN Publicaciones.

Gitman, L., \& Castro Gutiérrez, O. (2010). Administración Financiera. Decimoprimera Edición. México: Pearson Educación.

Herrera, L., Medina, A., \& Naranjo, G. (2004). Tutoría de la Investigación Científica. Quito: Diemerino Editores.

Niño Rojas, V. M. (2011). Metodología de la Investigación. Bogotá: Ediciones de la U.

Revista ENIAC Pesquisa, Guarulhos (SP), V.6, n.2, jun.- dez. 2017 
Olivares-Valentín, J. A. (Diciembre de 2011). Dialnet. Obtenido de Ejecución de la estrategia: Clave para el éxito empresarial: file://C:/Users/Samsung/Downloads/DialnetEjecucionDeLaEstrategiaClaveParaElExitoEmpresarial-4106559.pdf

Pascale, R. (2009). Decisiones Financieras. Sexta Edición. Buenos Aires: Prentice Hall Pearson Education.

Ramírez, D. N. (2013). Contabilidad Administrativa: Un enfoque estratégico para competir. En D. N. Ramírez, Contabilidad Administrativa: Un enfoque estratégico para competir (págs. 218-222). México: The McGraw-Hill Companies.

Rivero, J. (2001). Catedrático de Contabilidad de la Empresa y Estadisticas de Costes. En J. Rivero, Contabilidad Financiera (págs. 25-35). España: Trivium S.A.

Robbins, S. P., \& Coulter, M. (2010). Administración. En S. P. Robbins, \& M. Coulter, Administración: Planeación (págs. 144-147). México: Pearson Educación.

SEPS. (04 de 01 de 2016). Obtenido de Cooperativas Financieras Clasificadas por Segmentos:

https://www.google.com.ec/url?sa=t\&rct=j\&q=\&esrc=s\&source=web\&cd=1\&cad= rja\&uact $=8 \&$ sqi $=2 \& v e d=0$ ahUKEwjx04eavNPMAhWDkh4KHUH4CHsQFggaMA A\&url=http\%3A\%2F\%2Fwww.seps.gob.ec\%2Fdocuments\%2F20181\%2F341872 \%2FCOOPERATIVAS\%2BFINANCIERAS\%2BCLASIFICADAS\%2BPOR\%2BS EGMENTO

Superintendencia de Bancos. (s.f.). Golsario de Términos Financieros. Obtenido de http://www.superbancos.gob.ec/practg/sbs_index?vp_art_id=70\&vp_tip=2

Thompson, A. A., Peteraf, M. A., Gamble, J. E., \& Strickland, A. J. (2012). Administración Estratégica. México: McGraw-Hill Companies.

Warren, C., Reeve, J., \& Fess, P. (2005). Contabilidad Financiera. Novena Edición. México: Cengage Learninig. 
Zapata Sánchez, P. (2011). Contabilidad General. En Contabilidad General Con base a las NIIF (pág. 361). Colombia: McGraw Hill Interanericana.

Revista ENIAC Pesquisa, Guarulhos (SP), V.6, n.2, jun.- dez. 2017 
Anexo 1. Cálculos de los indicadores del patrimonio

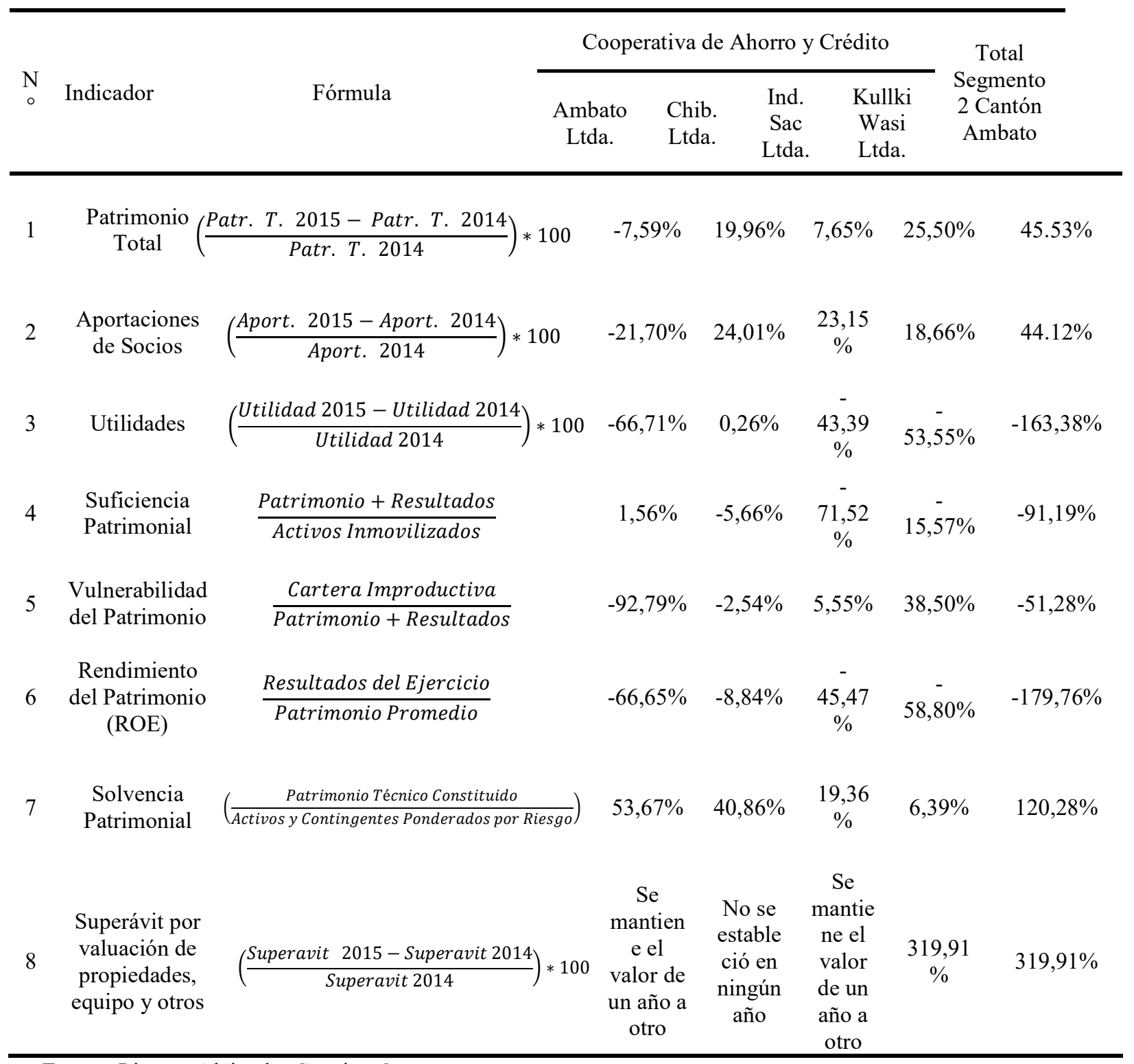

Fuente: Lissette Alejandra Gancino Ortega 